\title{
USO OU NÃO USO DO TORNIQUETE NA VIDEOARTROSCOPIA DO JOELHO: ESTUDO CLÍNICO MULTICÊNTRICO, PROSPECTIVO, DUPLO-CEGO E ALEATÓRIO
}

\author{
TOURNIQUET VERSUS NO TOURNIQUET USE IN KNEE VIDEOARTHROSCOPY: A \\ MULTICENTRIC, PROSPECTINE, DOUBLE-BLIND, RANDOMIZED CLINICAL TRIAL
}

Lúcio Honório de Carvalho Júnior', Luiz Fernando Machado Soares², Matheus Braga Jacques Gonçalves², Tiago Pinto de Oliveira Gomes ${ }^{3}$, Juraci Rosa de Oliveira ${ }^{4}$, Mauro Gualberto Coelho 5 , Rogério Ferreira Santos 6 , Marco Antônio Percope de Andrade 7 , Guilherme de Lima e Silva ${ }^{8}$, Eduardo Nilo Vasconcelos Novais ${ }^{8}$

\section{RESUMO}

Objetivo: Avaliar se com o uso da artrobomba (equipo de irrigação com sensor de pressão), o uso do torniquete pneumático poderia interferir no tempo cirúrgico, na recuperação de movimentos e no volume articular em pacientes submetidos à videoartroscopia do joelho para meniscectomia parcial. Métodos: Foram avaliados em cinco diferentes centros e com sete diferentes cirurgiões, 103 pacientes divididos de forma aleatória em dois grupos quanto à utilização ou não do torniquete pneumático. As variáveis foram avaliadas durante a cirurgia e com sete dias de pós-operatório. Resultados: Não se encontrou diferença estatisticamente significativa entre nenhuma das variáveis estudadas. Conclusão: Não existem razões que justifiquem ou desabonem o uso do torniquete nessa situação específica.

\section{ABSTRACT}

Objective: To evaluate if by using a pressure sensitive pump, tourniquet use could affect surgical time, range of motion recovery, and joint volume in patients who underwent knee arthroscopy for medial partial meniscectomy. Methods: Two groups of 103 patients operated in five different centers by seven different surgeons were evaluated after a random selection to use or not use a pneumatic tourniquet. The variables were evaluated during surgery and seven days post-operation. Results: No statistically significant differences were found among any of the variables studied. Conclusion: There is no reason to justify or disapprove tourniquet use in this specific situation.

Keywords - Surgery; Arthroscopy; Tourniquets

\section{INTRODUÇÃO}

O campo cirúrgico exangue é um dos grandes facilitadores da cirurgia, particularmente da ortopédica. $\mathrm{Na}$ videoartroscopia, sua obtenção é facilitada pelo uso do torniquete pneumático, que submete a raiz do membro a pressões duas a três vezes maiores do que a pressão sistólica, facilitando a visualização e a realização do procedimento, seja pela ausência, seja pela grande diminuição do sangramento ${ }^{(1,2)}$.

1 - Professor Adjunto Doutor do Departamento do Aparelho Locomotor da Faculdade de Medicina da UFMG. Membro do Grupo de Cirurgia do Joelho do Hospital Madre Teresa de Belo Horizonte, MG.

2 - Membro do Grupo de Cirurgia do Joelho do Hospital Madre Teresa de Belo Horizonte, MG.

3 - Especializando em Cirurgia do Joelho do Hospital Madre Teresa de Belo Horizonte, MG.

4 - Professor e Preceptor da Residência Médica em Ortopedia do Hospital Universitário São José de Belo Horizonte. Membro do Grupo de Cirurgia do Joelho da instituição.

5 - Preceptor da Residência Médica do Hospital Ortopédico de Belo Horizonte. Membro do Grupo de Cirurgia do Joelho do Hospital Madre Teresa de Belo Horizonte, MG.

6 - Médico Ortopedista do Hospital da Fundação Ouro Branco de Ouro Branco, Belo Horizonte, MG

7 - Professor Adjunto Doutor do Departamento do Aparelho Locomotor da Faculdade de Medicina da UFMG. Chefe do Serviço de Ortopedia e Traumatologia do Hospital das Clínicas da UFMG, MG.

8 - Membro do Serviço de Ortopedia do Hospital das Clínicas da UFMG, MG.

Trabalho realizado no Hospital Madre Teresa de Belo Horizonte, no Hospital Universitário São José de Belo Horizonte, no Hospital Ortopédico de Belo Horizonte, no Hospital da Fundação Ouro Branco de Ouro Branco e no Hospital das Clínicas da Universidade Federal de Minas Gerais.

Correspondência: Rua Olavo Carsalade Vilela, 264, Residencial Ipê da Serra - 34000-000 - Nova Lima MG. E-mail: luciohcj@medicina.ufmg.br 
Apesar do seu grande benefício, o uso do torniquete não é isento de riscos, pois a compressão traumatiza tecidos e diversas complicações são descritas quando de seu uso prolongado (principalmente após duas horas) ${ }^{(3)}$.

Mesmo em utilizações rápidas, a compressão muscular seria importante o suficiente para retardar a recuperação pós-operatória que seria dolorosa, pois o paciente necessitaria recuperar-se de duas agressões: além da cirúrgica também daquela produzida pelo uso do torniquete ${ }^{(4)}$.

Desde os trabalhos iniciais de Watanabe na década de 60, a artroscopia tem evoluído rapidamente, seja como método diagnóstico ou terapêutico. Sua utilização tem se expandido para as mais diversas articulações e o uso da microcâmera possibilitou, junto com o vídeo, grande melhora nas imagens obtidas ${ }^{(1)}$.

Para melhor visualização durante a videoartroscopia, foi desenvolvida e popularizada a partir do início da década de 90 a utilização de "artrobomba" (equipo de irrigação provido de sensor de pressão), que consegue controlar, além dessa última, o fluxo de líquido entrando e saindo da articulação ${ }^{(3)}$.

A meniscectomia artroscópica é tradicionalmente considerada como procedimento de baixa morbidade, com rápida recuperação pós-operatória. $\mathrm{O}$ uso do torniquete nesse procedimento apresenta literatura escassa e a relevância clínica do seu uso permanece indefinida, bem como sua influência no pós-operatório ${ }^{(4,5)}$.

O objetivo desse trabalho é avaliar se utilizando o equipo de irrigação com sensor de pressão, o uso do torniquete pneumático poderia influenciar o tempo cirúrgico, a recuperação de movimentos e o volume articular em pacientes submetidos à videoartroscopia do joelho para meniscectomia parcial.

\section{MATERIAL E MÉTODOS}

Foram avaliados 103 pacientes submetidos à videoartroscopias do joelho por sete diferentes cirurgiões em cinco diferentes centros, entre janeiro de 2006 e dezembro de 2007, divididos por sorteio em dois grupos:

Grupo $1(\mathrm{n}=51)$ : pacientes em que o torniquete pneumático, apesar de instalado na raiz da coxa do membro operado, não foi insuflado.

Grupo $2(\mathrm{n}=52)$ : pacientes em que o torniquete pneumático instalado na raiz da coxa foi insuflado com pressão de 350 milímetros de mercúrio.

Foram incluídos somente pacientes com lesões isoladas do menisco medial (degenerativa ou traumática) para as quais foi realizada meniscectomia parcial. Os critérios de exclusão foram: presença de outras lesões meniscais ou ligamentares no mesmo joelho ou no contralateral, doenças inflamatórias e lesões condrais para as quais fosse necessária qualquer intervenção que não sua simples regularização.

Todos os pacientes foram operados na posição supina após bloqueio anestésico epidural ou espinhal.

Nos dois grupos foi utilizado, para realização da videoartroscopia, "artrobomba", de diferentes marcas e modelos, reguladas para manter pressão intra-articular constante de 55 milímetros de mercúrio $(\mathrm{mmHg})$ e fluxo de 1,5 litros por minuto.

No momento do procedimento cirúrgico, foram coletados todos os dados relativos ao paciente e à cirurgia.

Todos os pacientes foram avaliados com sete dias de pós-operatório, sendo mensurados a perimetria articular a cinco centímetros do pólo superior da patela e a amplitude de movimento de cada joelho. Nessa avaliação, nem o paciente nem o examinador sabiam a que grupo o primeiro pertencia.

Os resultados foram analisados através do programa Epi-Info versão 6.0, utilizando o método de análise $t$ de Student para comparação de amostras pareadas. Foram ainda realizados os testes de Mann-Whitney/Wilcoxon (teste de Kruskal-Wallis).

O estudo foi aprovado pela Comissão de Ética em Pesquisa do Hospital Madre Teresa e posteriormente referendado pelas suas correspondentes nas demais instituições. Foram obtidos consentimentos por escrito de todos os pacientes.

\section{RESULTADOS}

Foram avaliados 103 pacientes sendo 41 mulheres e 62 homens. A média de idade foi de 49,22 anos, variando entre 15 e 81 anos. A média de idade no grupo 1 foi de 49,17 anos (variando entre 15 e 80 anos). No grupo 2 a média de idade foi de 49,26 anos (variando entre 20 e 81 anos). Não foi observada diferença estatística entre as idades dos dois grupos (teste $t$ com valor $p=0,9766 \mathrm{e}$ teste Kruskall-Wallis com valor $p=0,9055)$.

Em relação ao tempo cirúrgico nos dois grupos, o tempo médio no grupo 1 foi 21,29 minutos, variando entre oito e 60 minutos. No grupo 2 o tempo médio foi 21,71 minutos, variando entre oito e 45 minutos. Não foi observada diferença estatisticamente significante entre os dois grupos (teste $t$ de Student com valor $p=0,8528 \mathrm{e}$ teste de Kruskall-Wallis com valor $p=0,5743$ ).

Com sete dias de pós-operatório, a diferença entre a amplitude de movimento (ADM) no lado operado em relação ao lado não operado no grupo 1 foi, em média, 
8,36 graus, variando entre 0 e $50^{\circ}$. No grupo 2 , a média foi 8,70 graus, variando entre 0 e $50^{\circ}$. A comparação entre estes dois grupos pelo teste $t$ de Student mostrou valor $p$ $=0,8829$. Para as mesmas variáveis, o teste de KruskalWallis teve valor $p=0,3966$.

Em relação à perimetria articular com sete dias de pós-operatório no grupo 1 observou-se que a média de aumento de volume do lado operado foi $0,2686 \mathrm{~cm}$, variando entre $-2,5$ e $3 \mathrm{~cm}$. No grupo 2, a média de aumento de volume foi $0,2788 \mathrm{~cm}$, variando entre -2 e $4 \mathrm{~cm}$. Comparando-se esses dois grupos pelo teste $t$ de Student, obteve-se valor $p=0,9669$. Para as mesmas variáveis, o teste Kruskal-Wallis teve valor $p=0,9813$.

Os resultados encontram-se sumarizados na Tabela 1.

Tabela 1 - Comparação entre os grupos 1 e 2 e respectivo valor $p$

\begin{tabular}{c|c|c|c}
\hline & $\begin{array}{c}\text { Grupo 1 (sem } \\
\text { torniquete) }\end{array}$ & $\begin{array}{c}\text { Grupo 2 (com } \\
\text { torniquete) }\end{array}$ & Valor p \\
\hline Idade em anos & 49,1765 & 49,26 & 0,9766 \\
\hline $\begin{array}{c}\text { Tempo cirúrgico em } \\
\text { minutos }\end{array}$ & 21,2941 & 21,7115 & 0,8528 \\
\hline $\begin{array}{c}\text { Diferença de ADM } \\
\text { entre os joelhos em } \\
\text { graus }\end{array}$ & 8,70 & 8,36 & 0,8829 \\
\hline $\begin{array}{c}\text { Diferença de } \\
\text { perimetria entre } \\
\text { os dois joelhos em } \\
\text { centímetros }\end{array}$ & 0,2686 & 0,2788 & 0,9669 \\
\hline
\end{tabular}

Fonte: Serviço de Arquivo Médico das instituições.

\section{DISCUSSÃO}

Apesar da impressão inicial de melhor visualização com o uso do torniquete (já relatada por Kirkley et $a l^{(6)}$ ), tal fato não se mostrou relevante para o procedimento pois o tempo cirúrgico foi menor no grupo em que ele não foi utilizado.

O torniquete instalado na raiz do membro não foi insuflado em nenhum paciente do grupo 2 , mesmo que inicialmente o cirurgião tivesse alguma dificuldade na visualização. Sua colocação foi uma exigência da Comis-

\section{REFERÊNCIAS}

1. Dandy DJ Arthroscopy of the knee. London: Gower Medical Publishing; 1984 p.12-3.

2. Roberts TS, Terry R. Complications of knee surgery. In:. DeLee JC, Drez D Jr Orthopaedic sports medicine. Philadelphia: W.B. Saunders Company; 1994. p.1528-48.

3. Crenshaw AH. Campbell operative orthopaedics. St. Louis: Mosby; 1987.

4. Thorblad J, Ekstrand J, Hamberg P, Gillquist J. Muscle rehabilitation after arthroscopic meniscectomy with or without tourniquet control. A preliminary randomized study. Am J Sports Med. 1985;13(2):133-5.

5. Graf B, Jensen K, Orwin J, Duck H, Hagen P, Keene J. The effect of tourni- são de Ética alegando razões de segurança para o paciente que em uma eventual dificuldade que impedisse o prosseguimento da cirurgia poderia ter seu término sob compressão do membro, fato que o excluiria do trabalho.

Os achados e a pressão utilizada por Kirkley et $a l^{(6)}$ concordam parcialmente com os desse estudo. Apesar de usarem pressão de $300 \mathrm{mmHg}$, não encontraram nenhuma diferença entre dois grupos escolhidos de forma aleatória quanto a diversos quesitos, inclusive amplitude de movimentos, que também foi semelhante nos dois grupos. Apesar dos cirurgiões envolvidos estimarem que a visualização sem torniquete era três vezes pior do que com seu uso, de forma semelhante ao observado nesse trabalho o tempo cirúrgico médio foi semelhante entre os dois grupos (31,1 e 30,5 minutos, respectivamente).

O menor tempo cirúrgico médio dos dois grupos desse trabalho em relação ao relatado por Kirkley et $a l^{(6)}$, pode ser explicado pela padronização das lesões intra-articulares realizada no primeiro e não mencionada no segundo.

$\mathrm{Na}$ opinião dos autores o uso do equipo de irrigação com sensor de pressão foi fundamental para a realização do procedimento com campo exangue e sem que o torniquete fosse insuflado. Esse cuidado torna incomparáveis os resultados encontrados com aqueles observados por Olszewski et $a l^{(7)}$ que utilizaram fluxo gravitacional e solução de epinefrina para evitar que o torniquete fosse utilizado.

Apesar do aparente malefício que a compressão poderia provocar nos músculos da coxa, a distenção articular por si só também pode ser nociva, mesmo naqueles pacientes em que o torniquete não foi insuflado. Tal evento foi observado e relatado por Thorblad et $a l^{(4)}$ e posteriormente comentado por Johnson ${ }^{(8)}$ particularmente em relação ao torque do quadríceps.

\section{CONCLUSÃO}

$\mathrm{Na}$ videoartroscopia do joelho para tratamento da lesão do menisco medial desde que se utilize o equipo de irrigação com sensor de pressão, não há razão que justifique ou desabone o uso do torniquete pneumático.

quet use on postoperative strength recovery after arthroscopic meniscectomy. 1996;19(6):497-500.

6. Kirkley A, Rampersaud R, Griffin S, Amendola A, Litchfield R, Fowler P. Tourniquet versus no tourniquet use in routine knee arthroscopy: a prospective, double-blind, randomized clinical trial. Arthroscopy. 2000;16(2): 121-6.

7. Olszewski AD, Jones R, Farrell R, Kaylor K. The effects of dilute epinephrine saline irrigation on the need for tourniquet use in routine arthroscopic knee surgery. Am J Sports Med. 1999;27(3):354-6

8. Johnson DS. Letter to the editor. Am J Sports Med. 1998;26(1):150. 